\title{
Adipo Fascio Cutaneous Perforator Based, Shape Modified Radial Forearm Flap: Vascular Analysis of Perforators \& Its Clinical Applications in Head \& Neck Reconstruction
}

\author{
Ehtaih Sham1, Jaumie A. Masia1, Thyagaraj Jayaram Reddy² \\ ${ }^{1}$ Department of Plastic, Craniofacial \& Reconstructive Surgery, Hospital Sant Pau, University Autonoma De Barcelona, Barcelona, \\ Spain \\ ${ }^{2}$ Department of Plastic \& Reconstructive Surgery, Vydehi Institute of Medical Sciences, Bangalore, India \\ Email: ^ehtaihsham@yahoo.com,Jmasia@santpau.cat, raj.vani78@gmail.com
}

How to cite this paper: Sham, E., Masia, J.A. and Reddy, T.J. (2018) Adipo Fascio Cutaneous Perforator Based, Shape Modified Radial Forearm Flap: Vascular Analysis of Perforators \& Its Clinical Applications in Head \& Neck Reconstruction. International Journal of Otolaryngology and Head \& Neck Surgery, 7, 268-286. https://doi.org/10.4236/ijohns.2018.75028

Received: July 16, 2018

Accepted: August 31, 2018

Published: September 3, 2018

Copyright $\odot 2018$ by authors and Scientific Research Publishing Inc. This work is licensed under the Creative Commons Attribution International License (CC BY 4.0).

http://creativecommons.org/licenses/by/4.0/

\begin{abstract}
Resume of the study \& Background: Radial forearm free flap with all its present day modifications is the workhorse of soft tissue reconstruction. Although there are several advantages, it requires sacrifice of a major artery of forearm. Several modifications are described in harvesting a forearm flap. In order to achieve a reliable, safe flap harvest $\&$ design one must have a very clear understanding of radial artery perforators, relative to its distribution, territory \& flow. The purpose of this study is to determine the location, size \& vascular territory of the radial artery cutaneous perforators $\&$ to demonstrate application of shape modification of radial forearm free flap based on its distal \& proximal perforators in various head \& neck defects. Materials \& Methods: Anatomical Study: 12 fresh human cadavers \& 24 cadaveric forearms were dissected to determine the number, location, size \& vascular territory of radial artery perforator. The cutaneous territory of distally dominant perforators was analyzed using methylene blue injections \& three-dimensional computed tomographic angiogram to determine the vascular network. Clinical Study: 15 patients with various head neck defects following oncological resections were reconstructed with shape modified adipo-fascio cutaneous free forearm flap. All these patients were prospectively followed for donor site healing, motor \& sensory nerve deficit, function \& quality of life questioner for donor site assessment. Results: 12 fresh human cadavers \& 24 cadaveric forearms were dissected, and a total of 222 perforators were dissected for an average of 18.5 perforators per forearm. 118 were smaller than $0.5 \mathrm{~mm}$ in diameter $(53.15 \%) \&$ were not clinically significant. 104 perforators were
\end{abstract}


greater than $0.5 \mathrm{~mm}$ in diameter (46.84\%) \& were clinically significant. 127 perforators $(57.20 \%)$ were radially distributed \& 95 perforators $(42.79 \%)$ had ulnar distribution. 90 perforators $(40.54 \%)$ were identified on distal side (Radial styloid) \& 132 perforators (59.45\%) were identified on proximal side (Lateral epicondyle). Mean number of perforators, on radial side was 10.6 \& 7.9 on ulnar side; comparison of both using student t paired test gives a $\mathrm{P}$ value of 0.006 , which is significant. Comparison of mean number of perforators on the distal side was 7.5 \& proximal side was 11.0; Student Paired t test gives a $\mathrm{P}$ value of 0.003 , which was statistically significant. Comparison of mean diameter of perforators on Distal side (1.11) \& Proximal side (0.86), side using Student Paired t test gives a $\mathrm{P}$ value of 0.01 which was statistically significant. A chi square test was done to compare mean diameter of perforators on distal side, which was more than $1 \mathrm{~mm}(80 \%)$ \& less than $1 \mathrm{~mm}(20 \%)$ \& on proximal side more than $1 \mathrm{~mm}(35.6 \%)$ \& less than $1 \mathrm{~mm}(64.4 \%)$. Chi square value of 42.406 was obtained, degree of freedom value was $1 \& \mathrm{P}$ value of $<0.001$ was achieved which was found to be highly significant. Methylene blue injections demonstrated clusters both in proximal \& distal forearm \& also marked the cutaneous territory of flap. Three-dimensional computed tomographic angiography reveals a network of linking vessels found to communicate between adjacent perforators \& running parallel to radial artery. A total of 15 patients were reconstructed with shape modified radial forearm flap following oncologic resections. Wound healing in all 15 patients was good, with scar assessment faring better than traditional radial forearm flap. There was no sensory or motor nerve deficit. Although pedicle length was comparatively shorter in shape-modified flap, there was no problem in anastomosing to neck vessels. Conclusion: Increase in knowledge of vascular territory of radial artery perforators with regards to numbers, size, location, \& cutaneous territory can lead to expanded use of radial forearm flap based on either distal or proximal perforator alone. Shape modified technique for harvesting radial forearm flap allows primary closure of donor site. Donor site is better healed and shows a predicted pattern, which is functionally and aesthetically good.

\section{Keywords}

Vascular Anatomy, Angio Computed Tomography, Perforator Flap, Radial Artery, Reconstruction, Vascularization

\section{Introduction}

Knowledge of vascular anatomy has inevitably led to innovations in flap design $\&$ use in clinical arena. The evolution of random pattern flaps to facio-cutaneous flaps to myocutaneous flaps \& finally to the perforator flaps has followed a linear progression, largely because of pioneering vascular anatomical studies. The information derived from such kind of work has fuelled an evolution in flap designs \& its clinical applications. 
The ultimate goal of reconstruction is to match optimal tissue replacement with minimum donor site morbidity while maintaining function. Perforator flaps meet these goals \& are the result of over 30 yrs of evolution in flap refinement \& design.

Since the original description of radial forearm flap in 1978 by Yang \& Yuzhi [1] \& Timmons anatomical description in 1986, this versatile flap has found numerous applications in plastic \& reconstructive surgery [2]. Its value in head \& neck reconstruction \& upper \& lower extremity reconstruction is well documented. Nevertheless, its application as both retrograde flow pedicled island flap \& free flap has resulted in two major draw backs:

1) Donor site morbidity.

2) Sacrifice of radial artery.

A study detailing the location \& vascular territory of the radial artery perforators therefore becomes necessary to facilitate operative planning \& to allow safer flap harvest. A well described territorial outline of the consistently located, clinically significant perforators would be useful for operative decision making \& forms the basis for shape modified radial forearm harvest.

\section{Materials \& Methods}

Anatomical Study: To determine the location, size \& vascular territory of radial artery adipo-facio cutaneous perforators involving 12 fresh human cadavers \& 24 cadaveric forearms.

Need for the study: Anatomical studies defining the perforator vessel anatomy $\&$ distribution have been done before. However there are very few studies defining the location of perforator clusters or the cutaneous territory along the radial artery axis. A well defined territorial outline of the consistently located, clinically significant perforators would be useful for operative decision making of shape modified adipo-facio cutaneous radial artery perforator flap.

Inclusion criteria:

12 fresh human cadavers with normal radial artery anatomy were included in study.

Exclusion criteria:

1) Cadavers more than one month old.

2) Anomalies of radial artery.

Clinical study:

15 patients who underwent oncological resections were reconstructed with shape modified adipo-fascio cutaneous radial forearm free flap. All 15 patients were prospectively evaluated for donor site healing, scar, motor \& sensory function \& quality of life assessment.

Inclusion criteria:

1) Small to moderate defects requiring thin pliable flap for reconstruction.

Exclusion criteria:

1) Patients with systemic complications, who are not fit enough for a microvascular procedure. 
2) Large defects.

\section{Methodology}

Anatomical study: 12 fresh human cadavers \& 24 cadaveric forearms were dissected. All radial artery adipo-facio cutaneous perforators were analyzed for:

1) Number of perforators.

2) Orientation of perforators.

a) Radial distribution.

b) Ulnar distribution.

3) Size of perforators.

4) Cutaneous territory.

Upper extremity at the level of elbow \& the brachial artery was cannulated \& perfused with warm saline. This was continued until the backflow was clear. Subsequently the artery was injected with $20 \mathrm{cc}$ of microfil red \& the arm was stored in refrigerator at $0^{\circ} \mathrm{C}$ for $24 \mathrm{hrs}$. A total of 24 flaps were dissected to access the size, numbers, \& location of radial artery perforators. Two flaps were used for vascular injection studies using methylene blue \& lead oxide. An incision is made on the palmar aspect of forearm, extending from wrist crease to elbow. All perforators originating from radial artery are identified \& dissected. Parameters include; orientation of perforators, whether they arise on the radial side/lateral or on the ulnar side/medial, distance from radial styloid for distal perforators \& from the lateral epicondyle for the proximal perforators, external diameter (Artery) using calipers, cutaneous cluster pattern with methylene blue injections.

In order to determine the vascular cutaneous territory of a distally based radial artery perforator flap, two forearm flaps were dissected based on distal most dominant perforator near the radial styloid. The artery was cannulated \& injected with methylene blue dye to examine the territory of cutaneous staining. Injection of contrast material (Lead oxide) was then injected \& the specimen subjected to three-dimensional CT Scan with volume rendering function in order to access the vascular territory \& identify linking vessels communicating with adjacent perforators. The results were statistical analyzed with student $\mathrm{T}$ paired test \& Chi-Square test (Software: SPSS 17-Chicago Inc., SPSS version 22, IBM. Corp.).

Clinical operative procedure:

Radial artery course is marked with the help of Doppler. Skin pinch test is done to mark the dimensions of skin paddle. Approximately $2 \mathrm{~cm}$ of skin width in distal forearm and $3 \mathrm{~cm}$ of skin width in proximal forearm can be harvested \& closed primarily. This may slightly vary depending on the fat content in subcutaneous plain. Flap is harvested unto the origin of radial artery. Skin Island is marked and divided after visualizing the perforators to the skin. Suturing of these individual skin paddles together is done to give this flap the desired dimensions required to reconstruct the defect. After the harvest with its desired 
dimension and achieving the required pedicle length, donor site is closed primarily without the need for a skin graft.

\section{Results}

12 fresh human cadavers \& 24 forearm specimens, a total of 222 perforators were dissected for an average of 18.5 radial artery perforators per forearm (Table 1). Of the total 222 perforators dissected 118 were smaller than $0.5 \mathrm{~mm}$ in diameter (53.15\%) these were not clinically significant. 104 perforators were greater than 0.5 $\mathrm{mm}$ in diameter (46.84\%) these were clinically significant (Figure 1 ).

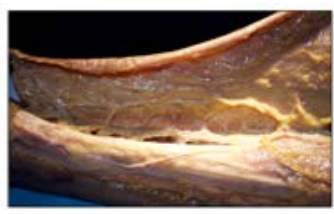

(a)

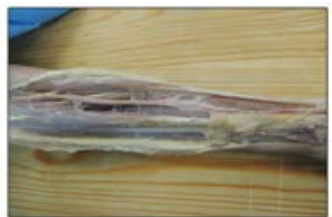

(a)

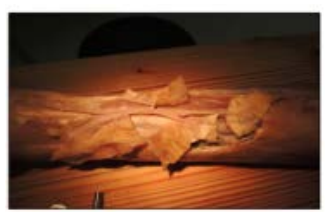

(a)

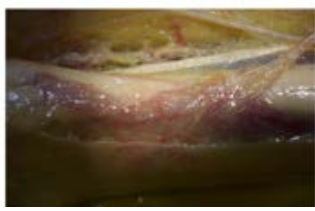

(b)

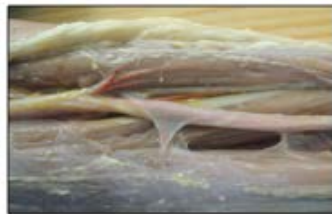

(b)

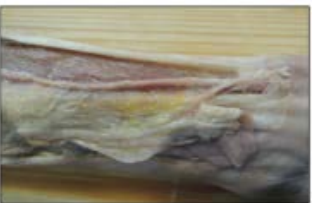

(b)

Figure 1. Radial artery perforators to skin.

Table 1. Master chart.

\begin{tabular}{cccccc}
\hline Cadavers & No of perforators & Radial side & Ulnar side & Distal side & Proximal side \\
\hline 1 & 19 & 10 & 9 & 8 & 11 \\
2 & 21 & 12 & 9 & 11 & 10 \\
3 & 20 & 11 & 9 & 7 & 13 \\
4 & 18 & 11 & 7 & 7 & 11 \\
5 & 12 & 10 & 2 & 7 & 5 \\
6 & 18 & 10 & 8 & 7 & 11 \\
7 & 20 & 13 & 7 & 6 & 14 \\
8 & 16 & 9 & 7 & 5 & 11 \\
9 & 19 & 10 & 9 & 10 & 9 \\
10 & 20 & 11 & 9 & 7 & 13 \\
11 & 21 & 9 & 12 & 9 & 12 \\
12 & 18 & 11 & 7 & 6 & 12 \\
& Total $=222$ & Total $=127$ & Total $=95$ & Total $=90$ & Total $=132$ \\
\hline
\end{tabular}


Of the 222 radial artery perforators dissected, 127 perforators (57.20\%) were radially distributed \& 95 perforators (42.79\%) had ulnar distribution (Table 1 ). A total of 90 perforators (40.54\%) were identified on distal side (Radial styloid) $\& 132$ perforators (59.45\%) were identified on proximal side (Lateral epicondyle) (Table 1). Mean number of perforators on radial side was $10.6 \& 7.9$ on ulnar side, a comparison of both using student $t$ paired test gives a $\mathrm{P}$ value of 0.006 , which was statistically significant (Table 2) (Figure 2). Comparison of mean number of perforators on the distal side was $7.5 \&$ proximal side was 11.0 , Student Paired $t$ test gives a $\mathrm{P}$ value of 0.003 , which was statistically significant (Table 3) (Figure 3). Comparison of mean Diameter of perforators between the Distal side (1.11) \& Proximal side (0.86), side using Student Paired t test gives a $\mathrm{P}$ value of 0.01 which was statistically significant (Tables 4-6) (Figure 4).

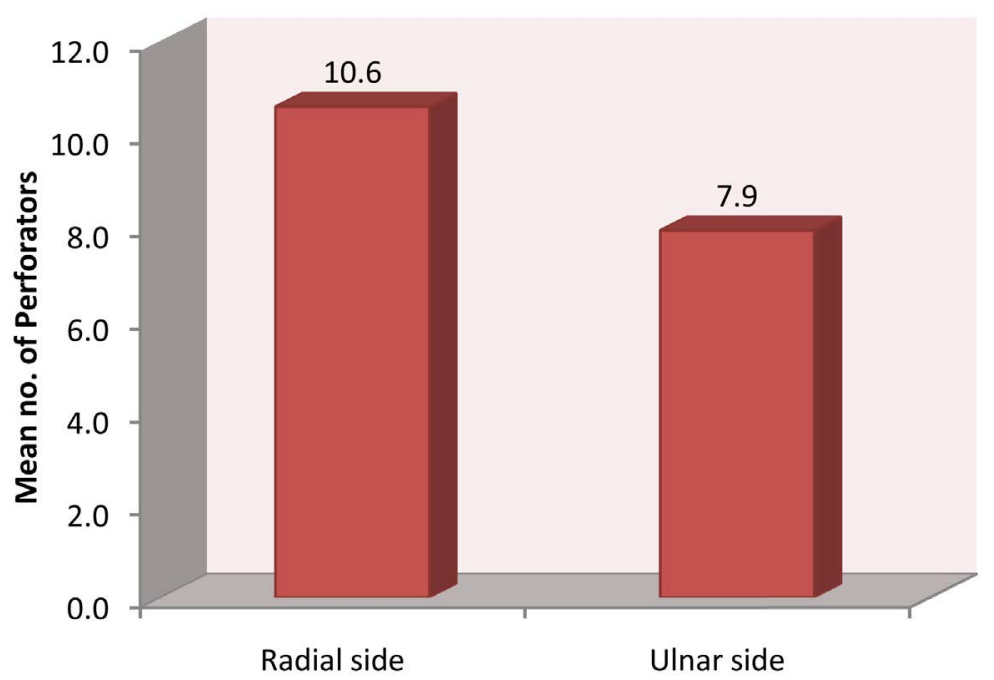

Figure 2. Comparison of mean number of perforators between radial and ulnar side.

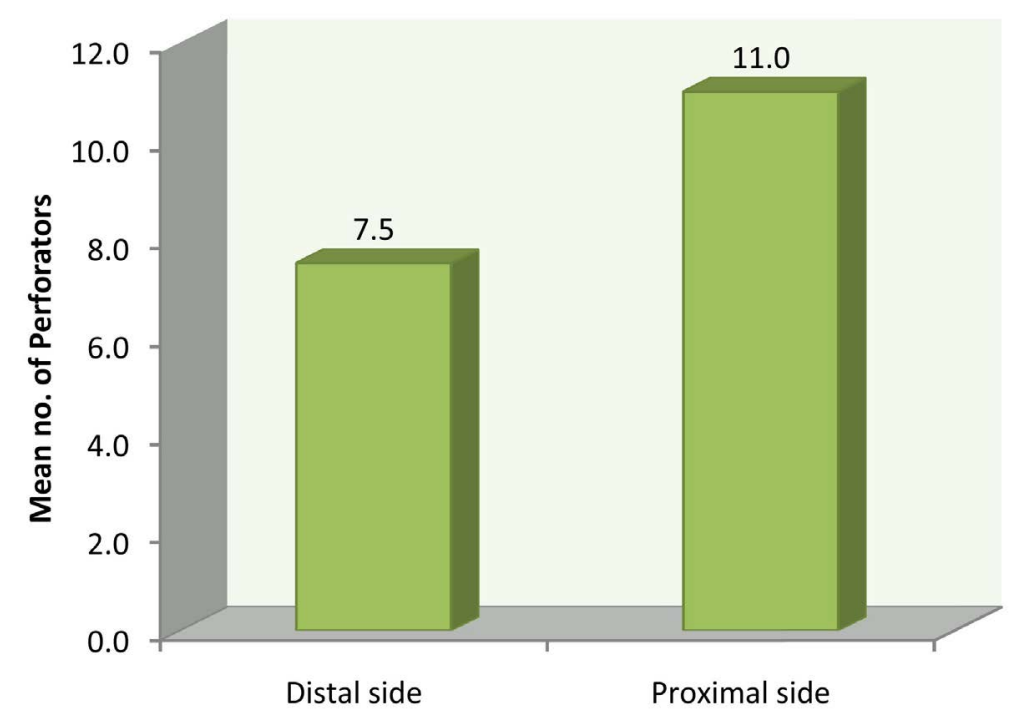

Figure 3. Comparison of mean number of perforators between the distal and proximal side. 


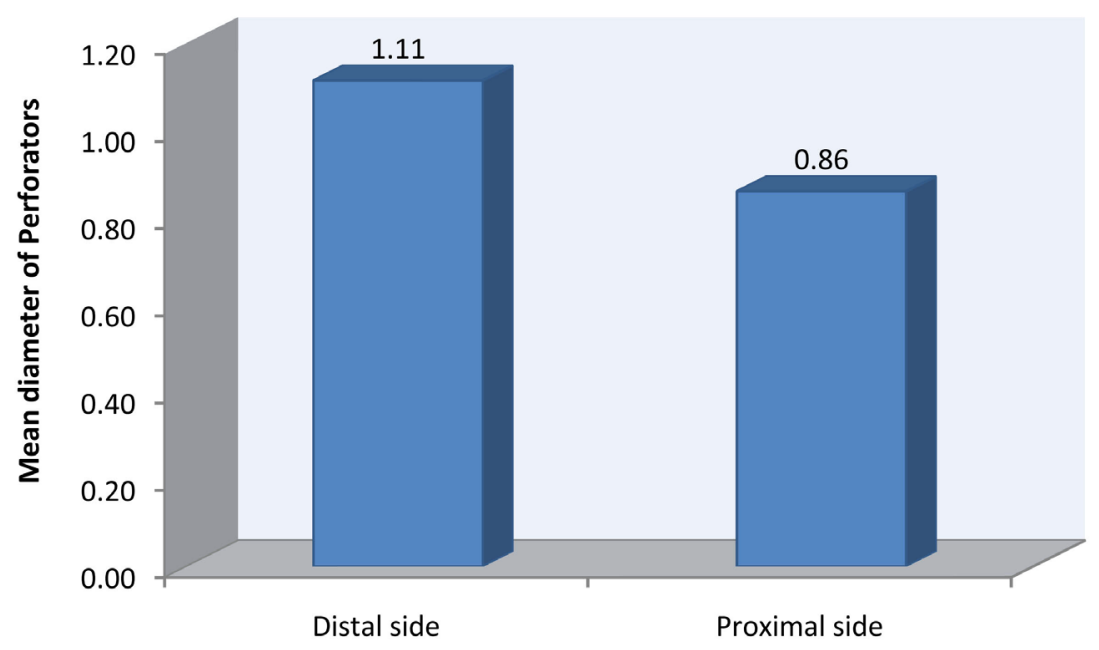

Figure 4. Comparison of mean diameters of perforators between the distal and proximal side.

Table 2. Comparison of mean number of perforators between the radial \& ulnar side-using student paired t test.

\begin{tabular}{cccccccccc}
\hline \multirow{2}{*}{ Sides } & $\mathrm{N}$ & Mean & SD & S.E.M & Mean Diff & $\frac{95 \% \text { CI of the Diff }}{2}$ tower & Upper & & P-Value \\
\hline Radial side & 12 & 10.6 & 1.2 & 0.3 & 2.7 & 0.9 & 4.4 & 3.370 & $0.006^{*}$ \\
Ulnar side & 12 & 7.9 & 2.4 & 0.7 & & & & & \\
\hline
\end{tabular}

Table 3. Comparison of mean number of perforators between the distal \& proximal side-using student paired t test.

\begin{tabular}{|c|c|c|c|c|c|c|c|c|c|}
\hline \multirow{2}{*}{ Sides } & \multirow{2}{*}{$\mathrm{N}$} & \multirow{2}{*}{ Mean } & \multirow{2}{*}{$\mathrm{SD}$} & \multirow{2}{*}{ S.E.M } & \multirow{2}{*}{$\begin{array}{c}\text { Mean } \\
\text { Diff }\end{array}$} & \multicolumn{2}{|c|}{$95 \%$ CI of the Diff } & \multirow{2}{*}{$\mathrm{t}$} & \multirow{2}{*}{ P-Value } \\
\hline & & & & & & Lower & Upper & & \\
\hline Distal side & 12 & 7.5 & 1.7 & 0.5 & \multirow{2}{*}{-3.5} & \multirow{2}{*}{-5.6} & \multirow{2}{*}{-1.4} & \multirow{2}{*}{-3.718} & \multirow{2}{*}{$0.003^{*}$} \\
\hline Proximal side & 12 & 11.0 & 2.3 & 0.7 & & & & & \\
\hline
\end{tabular}

Table 4. Mean diameter of perforators.

\begin{tabular}{lcc}
\hline & Distal (Radial Styloid) & Proximal (Lateral Epicondyle) \\
\hline 1 & 1.23 & 0.54 \\
2 & 1.01 & 1.00 \\
3 & 1.23 & 0.64 \\
4 & 0.92 & 0.63 \\
5 & 1.20 & 0.83 \\
6 & 1.17 & 0.92 \\
7 & 0.98 & 1.20 \\
8 & 0.87 & 1.00 \\
9 & 1.11 & 0.94 \\
10 & 1.20 & 0.68 \\
11 & 1.23 & 0.83 \\
12 & 1.22 & 1.11 \\
\hline
\end{tabular}


Table 5. Comparison of mean diameter of perforators between the distal \& proximal side-using student paired $t$ test.

\begin{tabular}{|c|c|c|c|c|c|c|c|c|c|}
\hline \multirow{2}{*}{ Sides } & \multirow{2}{*}{$\mathrm{N}$} & \multirow{2}{*}{ Mean } & \multirow{2}{*}{ SD } & \multirow{2}{*}{ S.E.M } & \multirow{2}{*}{ Mean Diff } & \multicolumn{2}{|c|}{$95 \%$ CI of the Diff } & \multirow{2}{*}{$\mathrm{t}$} & \multirow{2}{*}{ P-Value } \\
\hline & & & & & & Lower & Upper & & \\
\hline Distal & 12 & 1.11 & 0.13 & 0.04 & \multirow{2}{*}{0.25} & \multirow{2}{*}{0.08} & \multirow{2}{*}{0.43} & \multirow{2}{*}{3.132} & \multirow{2}{*}{$0.01^{*}$} \\
\hline Proximal & 12 & 0.86 & 0.21 & 0.06 & & & & & \\
\hline
\end{tabular}

Table 6. Mean number \& diameter on both sides.

\begin{tabular}{ccccc}
\hline Sides & Mean & Sides & Mean & Mean \\
\hline Radial side & 10.6 & Distal side & 7.5 & 1.11 \\
Ulnar side & 7.9 & Proximal side & 11.0 & 0.86 \\
\hline
\end{tabular}

A chi square test was done to compare mean diameter of perforators on distal side, which were more than $1 \mathrm{~mm}(80 \%)$ \& less than $1 \mathrm{~mm}(20 \%)$ \& on proximal side more than $1 \mathrm{~mm}(35.6 \%)$ \& less than $1 \mathrm{~mm}$ (64.4\%). Chi square value of 42.406 was obtained, degree of freedom value was $1 \& \mathrm{P}$ value of $<0.001$ was achieved which was found to be highly significant (Table 7) (Figure 5 and Figure 6). In almost all the dissected anatomical specimens at least one clinically significant perforator was found within $2 \mathrm{~cm}$ proximal to radial styloid (Figure 7). Methylene blue injections into the proximal part of radial artery demonstrated clusters both in proximal \& distal forearm \& also reveal a large flap cutaneous territory (Figure 7). Three-dimensional computed tomographic angiography (Figure 8) reveals a network of linking vessels found to communicate between adjacent perforators \& running parallel to radial artery. Large network of linking vessels could be found between fascia \& dermis, which also explains the ability to harvest forearm flap at the supra-fascial level.

In its clinical application 15 patients were reconstructed following oncological resections. The youngest being 31 yrs old \& oldest being 72 yrs of age (Figures 9-13). Total follow up period following reconstructions ranged between $6-12$ months. 13 patients were treated for head \& neck squamous cellcarcinoma \& one patient for basal cell carcinoma (Figure 10) \& one patient for oral sub-mucous fibrosis (Figure 13). Time taken for donor site healing was approximately 2 weeks, following which suture removal was done. In all 15 patients there was no sensory disturbance over the first web space on dorsum of hand \& motor function was good (Figure 14). Pedicle length though shorter, in all cases we managed to get a length of $8 \mathrm{~cm}$ to $10 \mathrm{~cm}$, which was sufficient enough for anatomizing to neck vessels. Scar assessment fared better (Figure 14). We developed a quality of life assessment score (total points 25) in which shape modified radial forearm flap showed better quality 23 (Score Table 8).

\section{Discussion}

Head \& Neck oncologists are often seem to confront with the difficult task of 
Distal $<1 \mathrm{~mm} \square$ Proximal $<1 \mathrm{~mm} \backsim$ Distal $>1 \mathrm{~mm}$ - Proximal $>1 \mathrm{~mm}$

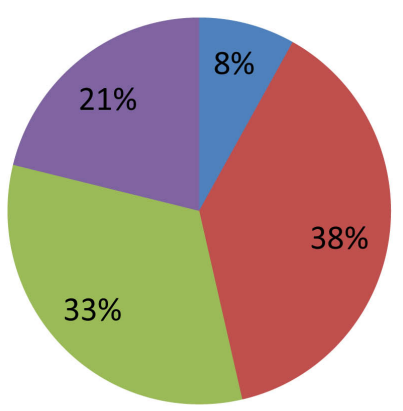

Figure 5. Pictorial presentation of chi square test.

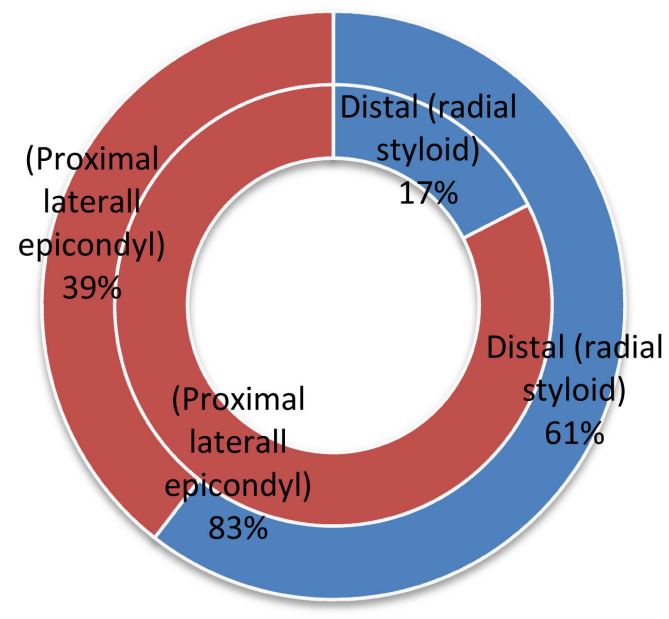

Figure 6. Component chart.

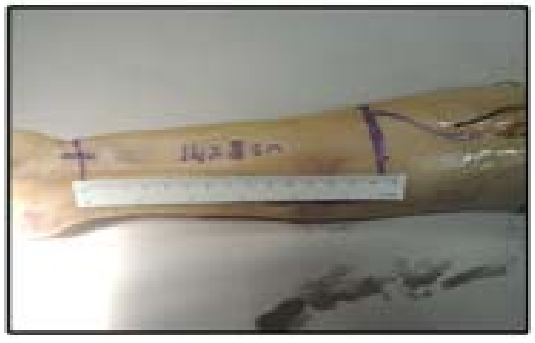

(a)

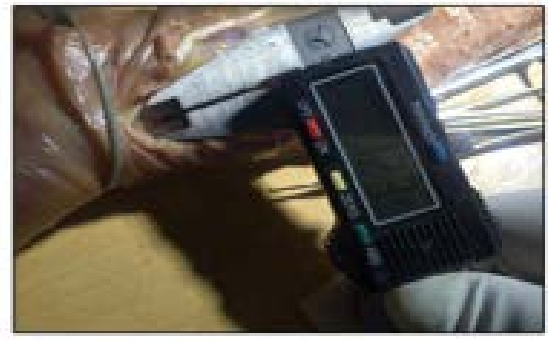

(c)

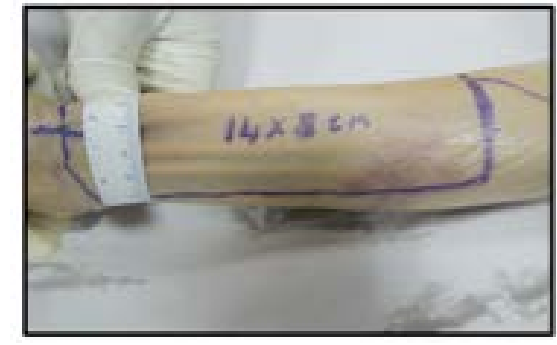

(b)

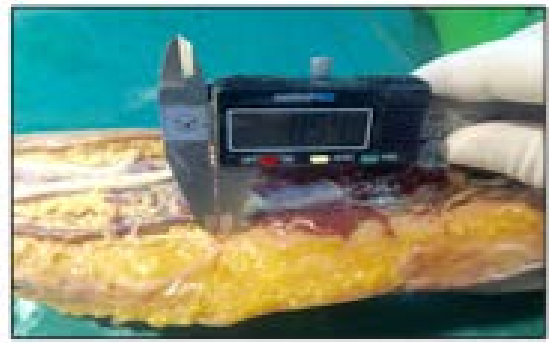

(d)

Figure 7. Cutaneous territory \& distal \& proximal perforators from radial artery. (a) Cutaneous territory of radial artery distal; (b) Perforators and maximum flap dimensions; (c) Distal perforator; (d) Procimal perforator. 


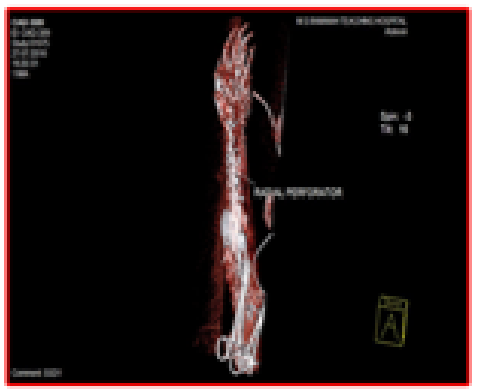

(a)

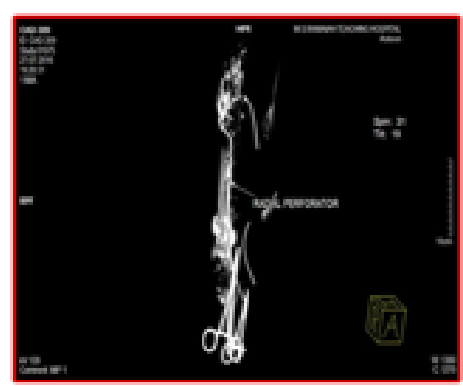

(b)

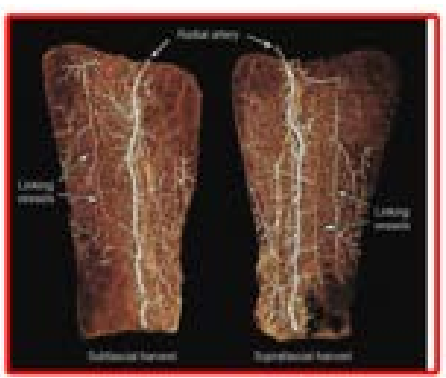

(c)

Figure 8. CT angiography of radial artery perforators.

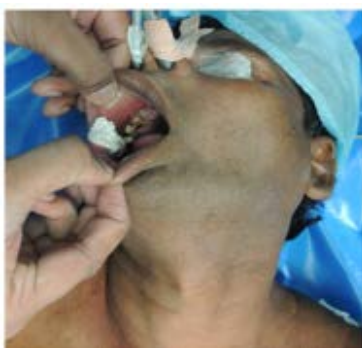

(a)

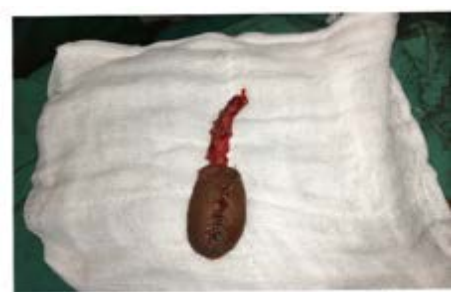

(c)

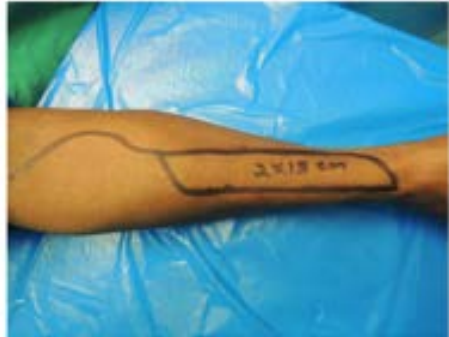

(b)

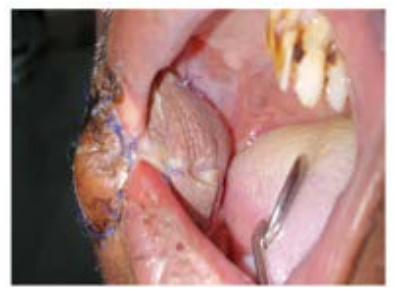

(d)

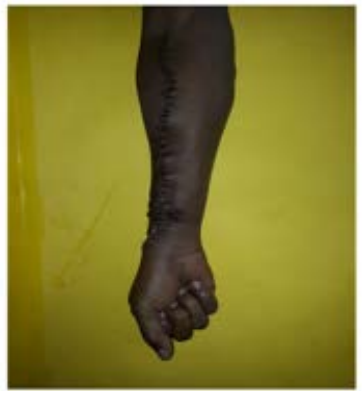

(e)

Figure 9. Case 1. 


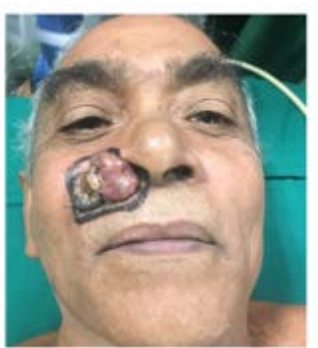

(a)

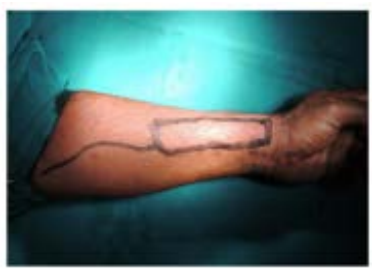

(c)

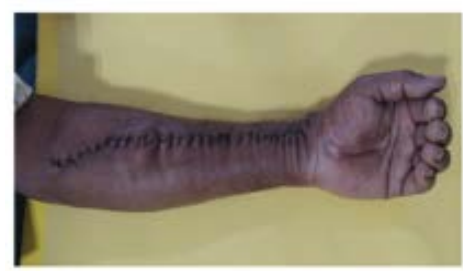

(e)

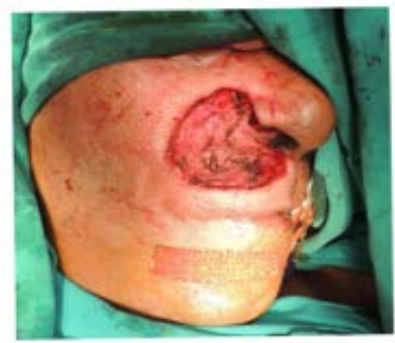

(b)

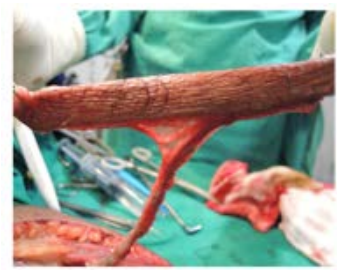

(d)

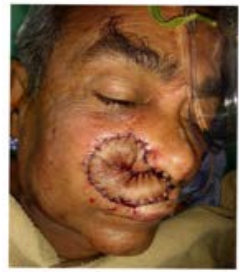

(f)

Figure 10. Case 2.

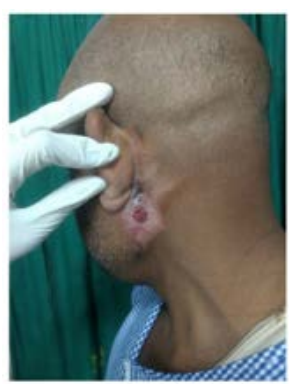

(a)

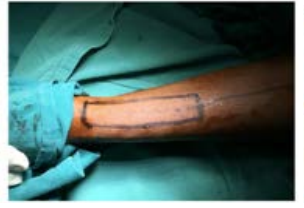

(c)

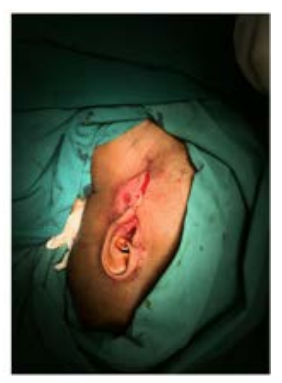

(b)

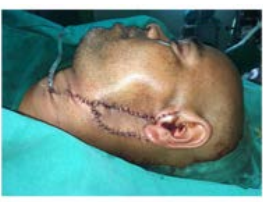

(d)

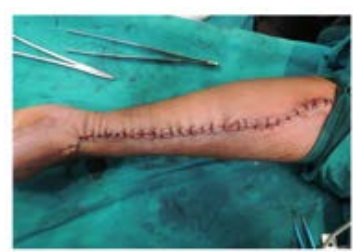

(e)

Figure 11. Case 3. 


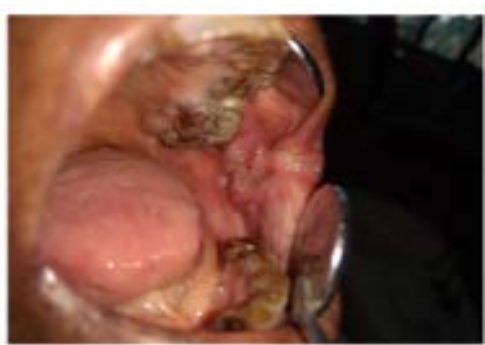

(a)

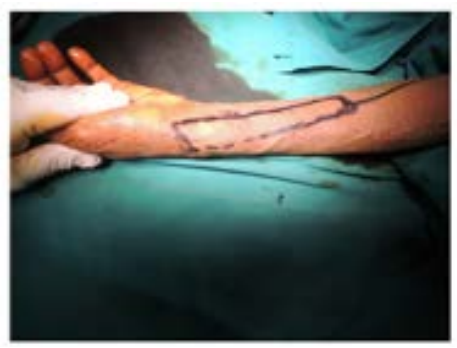

(c)

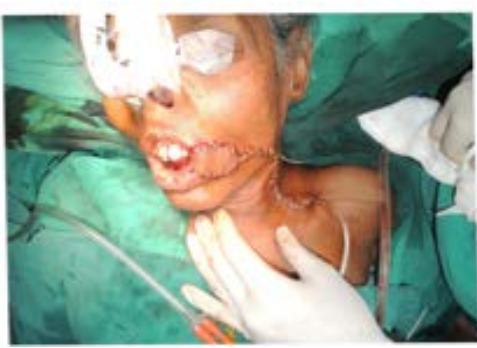

(e)

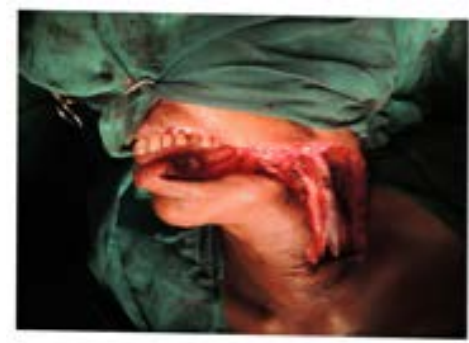

(b)

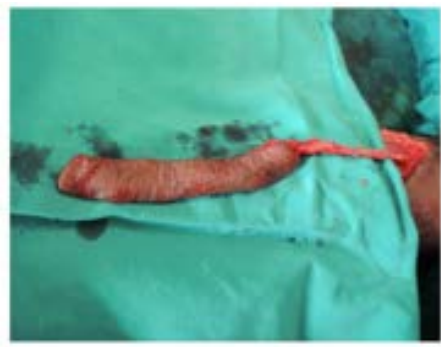

(d)

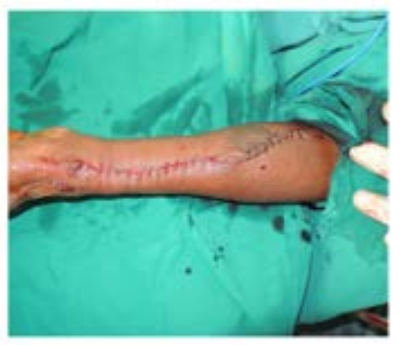

(f)

Figure 12. Case 4.

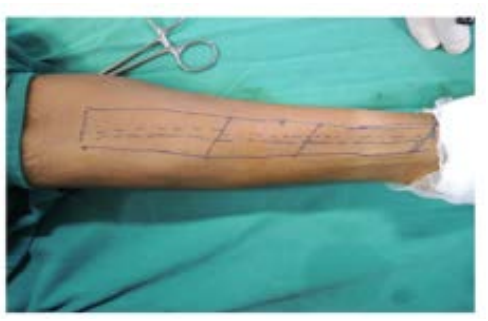

(a)

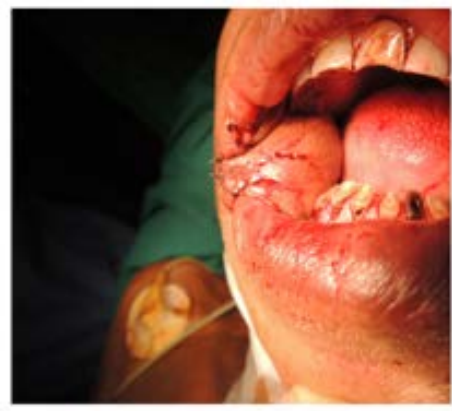

(c)

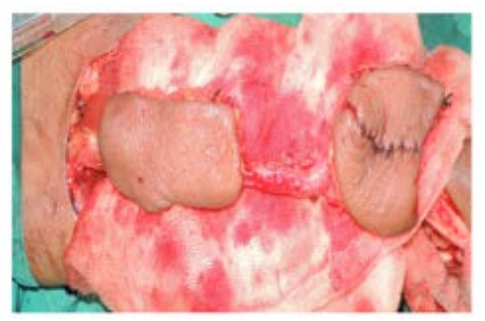

(b)

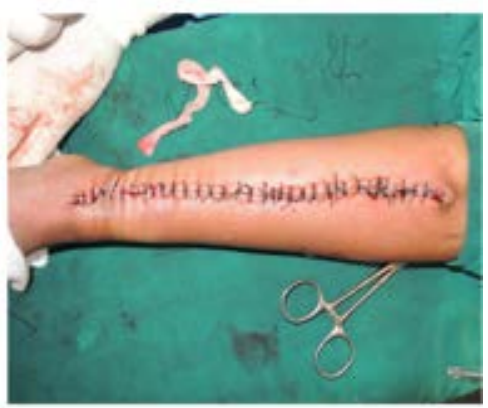

(d)

Figure 13. Case 5. 


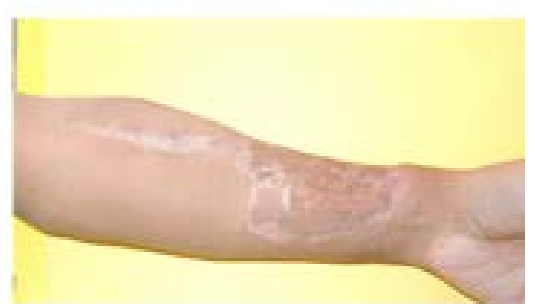

(a1)

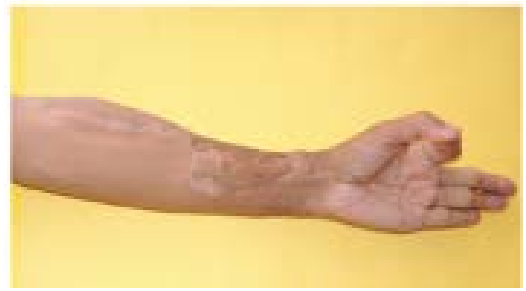

(b1)

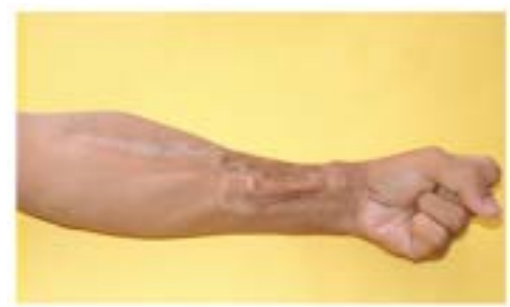

(c1)

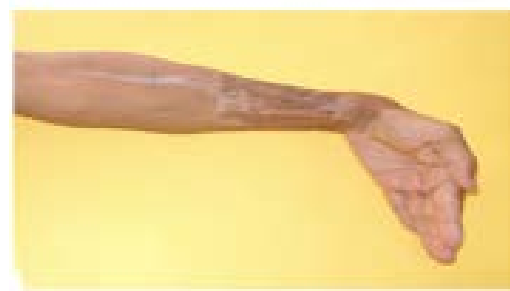

(d1)

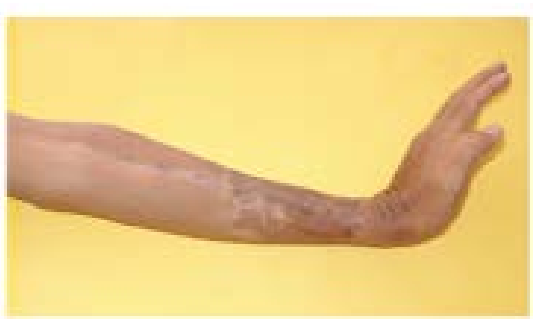

(e1)

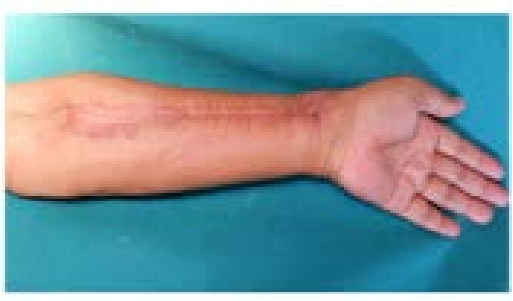

(a)

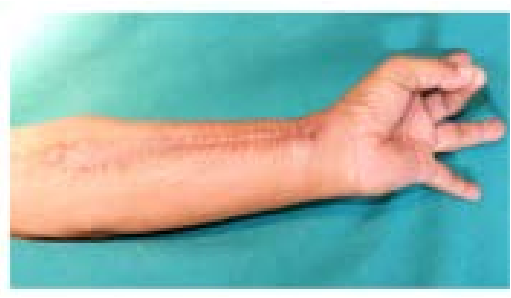

(d)

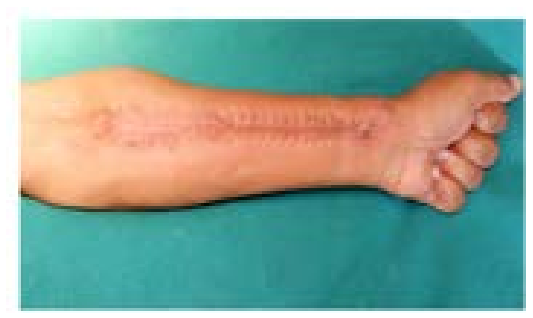

(c)

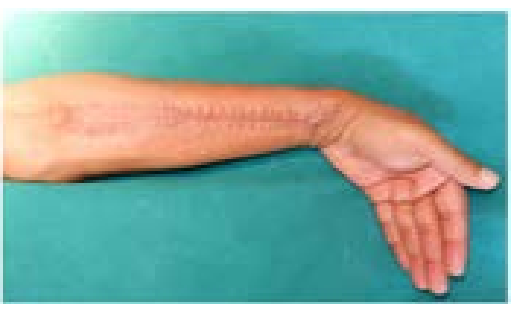

(d)

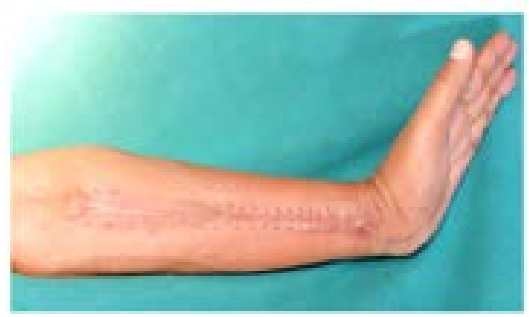

(e)

Figure 14. Donar site comparison of traditional radial forearm vs shaped modified radial forearm flap.

Table 7. Chi-Square table.

\begin{tabular}{cccccc}
\hline & Distal (radial styloid) & Proximal (lateral epicondyle) & $\mathrm{X}^{2}$ & $\mathrm{df}$ & P value \\
\hline$<1 \mathrm{~mm}$ & $18(20 \%)$ & $85(64.4 \%)$ & & & \\
$>1 \mathrm{~mm}$ & $72(80 \%)$ & $47(35.6 \%)$ & 42.406 & 1 & $<0.001$ \\
\hline
\end{tabular}


Table 8. Quality of life questioner.

\begin{tabular}{l} 
Question no \\
\cline { 2 - 6 } $\begin{array}{c}\text { average } \\
\text { Are you satisfied by this scar }\end{array}$
\end{tabular}

balancing cancer cure with preservation of function, cosmesis, \& quality of life when deciding best treatment protocols. Reconstruction in its true sense represents a big challenge for the reconstructive surgeon [3] [4].

Since the original description of radial forearm flap in 1978 by Yang \& Yuzhi, this versatile flap has found itself numerous applications in plastic \& reconstructive surgery [1] [2]. However its application as both retrograde flow pedicled island flap \& free flap has resulted in two major drawbacks drawing great attention among the reconstructive community [4] [5] [6].

1) Donor site morbidity in terms of esthetics, cold intolerance, slight to moderate functional deficit.

2) Sacrificing a major artery of forearm.

Several modifications \& innovations in terms of donor site closure, flap-harvesting techniques, and preservation of radial artery have been proposed \& practiced [7] [8] [9] [10] [11]. Increased knowledge of vascular anatomy has played a definitive role in increasing our ability to understand \& use perforator-based flaps for various clinical applications [12] [13].

As we gradually shift towards perforator based flap reconstructions, knowledge of individual perforators should supersede that of source artery [14]. Many surgeons have reported use of perforator flaps from forearm with ease, success, \& good functional outcome [15] [16] [17] [18]. However it is difficult to clinically establish the exact location of dominant radial artery perforator with a hand held Doppler because of superficial course of radial artery [19] [20]. In order to establish the basis for perforator flaps an anatomical study to determine the exact location \& vascular territory becomes all the more essential. In our present study we have investigated the location, size \& vascular pattern \& territory of radial artery in 12 fresh human cadavers involving 24 forearm specimens (Figure 1 \& Figure 7). We have demonstrated a total of 222 perforators with an average of 18.5 perforators per forearm. Perforators smaller than $0.5 \mathrm{~mm}$ were not considered as clinically suitable. Our study confirms a strong relation between the number \& diameter of perforators [16] [17] [20]. Distal side demon- 
strates less number of perforators but a large caliber perforator is consistently present within $2 \mathrm{~cm}$ of radial styloid in all of the 12 cadavers (Figure 7), this finding is found to be consistent with the previous anatomical studies on radial artery [15]. Michel Saint-Cyr et al. conducted an anatomical study in 26 fresh human cadaveric forearms to study \& determine the location, orientation, external diameter \& vascular territory of radial artery cutaneous perforators. The authors concluded that there are two main clusters of clinically significant perforators \& increased knowledge of size, location \& cutaneous territory of radial artery perforators can lead to expanded use of radial artery forearm flap based on these cutaneous perforators alone, without sacrificing the main radial artery [20].

In both distal \& proximal forearms, perforators arising from the main vessel have both radial \& ulnar distribution [20] (Figure 1 and Figure 7). These anatomical characteristics suggest an element of safety when harvesting flaps based on either distal or proximal perforator [20]. Our study demonstrates clusters of perforators in both proximal \& distal aspect of forearm [13] [14] [20].

Clinical use of perforator based radial artery free flap may have limited applications but nevertheless cases have been reported in literature [21]. The vascular anatomy of the fascia \& subcutaneous tissue of the distally based flaps have been well described [18]. The blood supply to the fascio-cutaneous flap is found to originate from 5 to 10 septo-cutaneous perforators arising from radial artery in the anatomical snuffbox [14] [15]. Anatomical \& clinical investigation of the radial forearm adipo-fascial flap based on distal perforators has been well described by Hamdy El-Khatib, who reported an anatomic study in eleven fresh \& fixed cadavers, designing an island adipofascial flap based on distal five to eight septo-cutaneous perforators of radial artery $\&$ their vena commitantes, great advantage being preservation of radial artery [13]. Three-dimensional computed tomographic angiography in our study has shown a comparable vascular territory with linking vessels found to communicate between adjacent perforators \& running parallel to radial artery [20] (Figure 8). Perforators travel to dermis \& form a network of linking vessels found between fascia \& dermis. This finding is consistent as reported by previous similar studies [13] [15]. Mark Schaverien conducted a study involving 12 fresh human cadavers \& 24 radial forearm flaps harvested from these cadavers to elucidate the role of deep fascia in perfusion of radial forearm flap. The authors concluded that inclusion of deep fascia during flap harvest does not contribute to flap perfusion or vascularity [18]. Although there are several anatomical study reports on vascular anatomy of radial artery, our study demonstrates the cutaneous territory \& location of clusters in detail. The results obtained from our study are clinically important in planning the exact dimension of the flap based on distal third perforators of radial artery. Perhaps the most significant finding in this study is the location of cutaneous perforators. Knowledge of these perforators can greatly facilitate the reconstructive surgeon in safe flap harvest \& design [21] [22]. Both distal \& proximal perforators can be used in designing of potential flaps for various reconstructive needs 
[15]. Three-dimensional computed tomographic angiogram confirms that suprafacial dissection does not compromise blood supply of the radial forearm pedicle perforator flap [9] [11] (Figure 8). The supply to the skin is ensured by means of multiple perforators originating from the radial \& ulnar sides of the radial artery, travelling to the skin \& communicating with each other in the subcutaneous tissue plane by multiple linking vessels. Each perforator has its own vascular territory, called a perforasome, which carries a multidirectional flow pattern that is highly variable \& complex. These perforasomes are linked to one another by both direct $\&$ indirect linking vessels, which themselves are linked by communicating branches. These numerous branches confer further protection form ischemia \& vascular injury \& explains the survival of single dominant perforator based flaps [20].

Linking vessels allow communication with adjacent perforasomes \& follow a direction that is parallel to the direction of perforator flow. Therefore, perforator flap skin paddles should be parallel to the linking vessel orientation, which makes it possible to harvest large flaps based on single dominant perforator [20] [23]. If the radial forearm flap donor-site defect could be repaired primarily, then hand rehabilitation could be initiated earlier and the overall complication rate would be lower, compared with the traditional technique using a split-thickness skin graft to close the defect (Figure 14). This has led many surgeons to search for alternate methods of harvesting this flap allowing primary closure of the donor site. These include procedures incorporating tissue expanders or rotation flaps. Mateev et al. suggested a novel way of harvesting radial forearm flaps, which was termed "Shape modified method". The series being reported for scalp reconstruction [19]. No such reports are available for reconstruction of head and neck defects even though the methods appear to be elegant and avoids the use of skin grafts. The major advantage of this technique is its ability to reconstruct donor defects primarily in a one-stage operation, with better aesthetic contours of the forearm. In comparison with traditional methods such as split thickness skin grafting, the overall complication rate is minimized because hand motion can be allowed earlier, with the flexor tendons sliding beneath the flap. Soutar et al. reported $28 \%$ of problems in extension of wrist with traditional method [5]; we have no such reports in modified groups because of primary closure in the later group. Studies by Fenton et al. reports specifically the aesthetic problems in traditional method of radial forearm flap [24]. None of our patients had any sensory nerve disturbance associated with radial nerve branch. (Dorsum of thumb and first web space) (Figure 14). Our shape modified radial forearm flap has fared much better when compared to other methods of direct closure of donor sites. Ching-Hua et al. used ulnar based bilobed flaps but found the final defect has a lengthy and unsightly scar [24] [25]. Elliot et al. used V-Y advancement for primary closure but could only harvest $4 \times 4 \mathrm{~cm}$ flaps and Berge et al. used tissue expanded skin but found difficult and used two stages for primary-Closure [26] [27]. We had problems with our method, which included reduction in pedicle length whenever the size of flap is increased. Maxi- 
mum pedicle length we could harvest was $10 \mathrm{~cm}$. Another probable problem mentioned in literature is the compression of the forearm due to tight closure. We did not have this complication in any of our 15 patients.

\section{Conclusion}

Each perforator holds a unique vascular territory. Vascular supply chain among perforators is highly complex \& both direct \& indirect linking vessels play a major role in maintaining flap perfusion. Our study demonstrates a consistent course of radial artery with clusters originating on both distal \& proximal ends, with each end becoming a pivot point for a pedicled flap rotation. Each perforator has the potential to become either a free or a pedicled flap depending on the size. In addition preservation of radial artery $\&$ ability of supra-facial harvest further lessens the morbidity burden. This phenomenon allows a myriad of perforator flap designs that can be tailored to match reconstructive defects. Present day reconstructive surgeon has more options \& alternatives in replacing like with like. Local flap alternatives become more plentiful \& flap design is limited only by the availability of clinically relevant perforators close to the defect. Free style perforator flap alternatives are limited by the size \& length of their respective source arteries \& veins.

\section{Conflicts of Interest}

The authors declare no conflicts of interest regarding the publication of this paper.

\section{References}

[1] Yang, G., Chen, B., Gao, Y., Liu, X., Li, J., Jiang, S. and He, S. (1981) Forearm Free Skin Flap Transplantation. National Medical Journal of China, 61, 139-141.

[2] Timmons, M.J., Missotten, F.E.M., Poole, M.D. and Davies, D.M. (1986) Complications of Radial Forearm Flap Donor Sites. British Journal of Plastic Surgery, 39 176-178. https://doi.org/10.1016/0007-1226(86)90078-0

[3] Mühlbauer, W., Herndl, E. and Stock, W. (1982) The Forearm Flap. Plastic and Reconstructive Surgery, 70, 336-342. https://doi.org/10.1097/00006534-198209000-00007

[4] Soutar, D.S. and McGregor, I.A. (1986) The Radial Forearm Flap in Intra-Oral Reconstruction. The Experience of 60 Consecutive Cases. Plastic and Reconstructive Surgery, 78, 1-8. https://doi.org/10.1097/00006534-198607000-00001

[5] Song, R., Gao, Y., Song, Y., Yu, Y. and Song, Y. (1982) The Forearm Flap. Clinics in Plastic Surgery, 9, 21-26.

[6] Lovie, M.J., Duncan, G.M. and Glasson, D.W. (1984) The Ulnar Artery Forearm Flap. British Journal of Plastic Surgery, 37, 486-492. https://doi.org/10.1016/0007-1226(84)90136-X

[7] Chang, S.C., Miller, G., Halbert, C.F., Yang, K.H., Chao, W.C. and Wei, F.C. (1996) Limiting Donor Site Morbidity by Suprafacial Dissection of the Radial Fore Arm Flap. Microsurgery, 17, 136-140. https://doi.org/10.1002/(SICI)1098-2752(1996)17:3<136::AID-MICR7>3.0.CO;2-K 
[8] Partecke, B.D., Buck-Gramcko, D. and Pachnucki, A. (1986) Use of a Fascia Flap of the Forearm in Soft Tissue Defects of the Extremities. (German) Handchir Mikrochir Plast Chir, 18, 353-355.

[9] Liang, M.D., Swartz, W.M. and Jone, N.F. (1994) Local Full Thickness Skin Graft Coverage for Radial Forearm Flap Donor Site. Plastic and Reconstructive Surgery, 93, 621-625. https://doi.org/10.1097/00006534-199493030-00032

[10] Webster, H.R. and Robinson, D.W. (1995) The Radial Forearm Flap without Fascia \& Other Refinements. European Journal of Plastic Surgery, 18, 11-13. https://doi.org/10.1007/BF00183691

[11] Wolf, K.D., Ervens, J. and Hoffmeister, B. (1995) Improvement of Radial Forearm Donor Site by Prefabrication of Facial Split Thickness Skin Grafts. Plastic and Reconstructive Surgery, 98, 358-361. https://doi.org/10.1097/00006534-199608000-00025

[12] Mahmoud, W.H. (2015) Radial Forearm Flap versus Radial Adipofascial Perforator Based Flap for Reconstruction of Hand Soft Tissue Defects. Donnish Journal of Medicine \& Medical Sciences, 2, 19-25.

[13] Rath, T., Millesi, W., Millesi-Schobel, G., Lang, S., Glaser, C. and Todoroff, B. (1997) Mucosal Prelaminated Flaps for Physiological Reconstruction of Intra Oral Defects after Resection. British Journal of Plastic Surgery, 50, 303-307. https://doi.org/10.1016/S0007-1226(97)90537-3

[14] El-Khatib, H. and Zeidan, M. (1997) Island Adipofascial Flap Based on Distal Perforators of the Radial Artery: An Anatomic \& Clinical Investigation. Plastic and Reconstructive Surgery, 100, 1762-1766. https://doi.org/10.1097/00006534-199712000-00018

[15] Yang, D.P., Moris, S.F., Tang, M.L. and Geddes, C.R. (2003) Reversed Forearm Island Flap Supplied by the Septocutaneous Perforator of the Radial Artery: Anatomical Basis \& Clinical Applications. Plastic and Reconstructive Surgery, 112, 1012. https://doi.org/10.1097/01.PRS.0000076192.14536.90

[16] Tiengo, C., Macchi, V., Porzionato, A., Stecco, C., Parenti, A., Bassetto, F. and De Caro, R. (2004) Anatomical Study of Perforator Arteries in the Distally Based Radial Forearm Fasciosubcutaneous Flap. Clinical Anatomy, 17, 636-642. https://doi.org/10.1002/ca.20009

[17] Schaverien, M. and Saint-Cyr, M. (2008) Suprafacial Compared with Subfacial Harvest of the Radial Forearm Flap: An Anatomic Study. Journal of Hand Surgery, 33A, 97-101.

[18] Mateev, M.A., et al. (2009) Shape Modified Radial Artery Perforator Flap Method. Analysis of 112 Cases. Plastic \& Reconstructive Surgery, 123, 1533-1543.

[19] Saint-Cyr, M. (2010) The Radial Artery Pedicled Perforator. Plastic and Reconstructive Surgery, 125, 1469-2010.

[20] Lin, J.-Y., Cheng, M.-H., Wei, F.C., Song, D. and Huang, W.-C. (2006) Proximal Forearm Flap Based on a Septocutaneous Vessel Form the Radial Artery. Plastic \& Reconstructive Surgery, 117, 955-960.

[21] Tiengo, C., Macchi, V., Porzionato, A., Stecco, C., Parenti, A., Bassetto, F. and De Caro, R. (2007) The Proximal Radial Artery Perforator Flap (PRAP-Flap): An Anatomical Study for Its Use in Elbow Reconstruction. Surgical and Radiologic Anatomy, 29, 245-251. https://doi.org/10.1007/s00276-007-0203-7

[22] Ciuman, R. and Dost, P. (2012) The Fore Arm Free Flap-Indications, Appropriate Selection, Complications \& Functional Outcomes. http://www.intechopen.com 
[23] Giordano, L., Bondi, S., Ferrario, F., Fabiano, B. and Bussi, M. (2012) Radial Forearm Free Flap Surgery: A Modified Skin Closure Technique Improving Donor Site Aesthetic Appearance. Acta Otorhinolaryngologica Italica, 32, 158-163.

[24] Fenton, O.M. and Roberts, J.O. (1985) Improving the Donor Site of the Radial Forearm Flap. British Journal of Plastic Surgery, 38, 504. https://doi.org/10.1016/0007-1226(85)90009-8

[25] Hsieh, C.-H. and Kuo, Y.-R. (2004) Primary Closure of Radial Forearm Flap Donor Defects with a Bilobed Flap Based on the Fasciocutaneous Perforator of the Ulnar Artery. Plastic and Reconstructive Surgery, 113, 1355-1360. https://doi.org/10.1097/01.PRS.0000112742.51430.A7

[26] Bardsley, A.F., Soutar, D.S., Elliot, D., et al. (1990) Reducing Morbidity in the Radial Forearm Flap Donor Site. Plastic and Reconstructive Surgery, 86, 287-292. https://doi.org/10.1097/00006534-199008000-00014

[27] Bergé, S.J. and Wiese, K.G. (2001) Tissue Expansion Using Osmotically Active Hydrogel Systems for Direct Closure of the Donor Defect of the Radial Forearm Flap. Plastic and Reconstructive Surgery, 108, 1-5.

https://doi.org/10.1097/00006534-200107000-00001 